\title{
Maternal And Perinatal Outcome in Eclampsia in Siddhartha Medical College, Govt. General Hospital, Vijayawada, Andhra Pradesh
}

\author{
${ }^{1}$ Dr. G.Chandrakala,MS (OBG), ${ }^{2}$ Dr. M.Tripurasundari,MD, DGO \\ ${ }^{1}$ Assistant Professor $O B G, S M C$ \\ ${ }^{2}$ Professor and HOD Deportment of $O B G, S M C$, Vijayawada, AP
}

\begin{abstract}
Objectives: To assess maternal and perinatal outcome in patients presenting with eclampsia.

Methods and Material: This is a prospective study done at Siddhartha Medical College, Government General Hospital, Vijayawada from April 2015 to March 2016. In all patients with eclampsia, maternal outcome was measured in terms of complications like pulmonary edema, hematological disorders, renal failure, cerebral haemorrhage and maternal death. Perinatal outcome was noted in terms of pre-term births, IUGR still births, NICU admissions.

Result: In 6880 deliveries in our hospital, 74 cases were diagnosed as eclampsia, giving an incidence of 10.7 per 1000 deliveries. There were two maternal deaths, giving a case fatality rate of 2.7\%.Eclampsia was commonest among the age group of 18-23 years. Primi Gravidae constituted $78.3 \%$ of the cases. Majority of patients (81.08\%) were unbooked. Antepartum eclampsia was seen in $67.5 \%$ of the cases more than Intrapartum $(10.8 \%)$ and postpartum (21.6\%). Forty-two patients had severe hypertension on admission diastolic BP >110 $\mathrm{mmHg}$. Thirty-two patients had mild hypertension diastolic BP $(90$ to $<110 \mathrm{mmHg})$. Forty $(54 \%)$ were delivered through caesarean section, twenty-five (33.3\%) had normal vaginal delivery and nine (12\%) had assisted vaginal delivery. Maternal complications include 3 cases of acute renal failure, 3 cases of HELLP syndrome, 2 cases of pulmonary edema, 2 cases of coagulation failure, 6 cases of abruption, 1 case of cortical blindness and 1 case of cerebral venous sinus thrombosis. Sixty-two babies were delivered live while twelve still births were recorded. Sixteen babies (21.6\%) had low birth weight and twenty-two babies (29.7\%) had normal birth weight. Conclusion: Eclampsia still remains a major cause of maternal morbidity and mortality. More awareness and enabling factors should be created for more women to access antenatal facilities. Information about danger signs of pre-eclampsia/eclampsia should be made available to antenatal cases.
\end{abstract}

Keywords: Eclampsia, perinatal, maternal complications.

\section{Introduction}

Eclampsia was the leading cause of maternal mortality. This is variously attributed to cultural practices leading to early child bearing in the wake of poor maternity care services that are grossly underutilized. Pre-eclampsia and eclampsia are major causes of maternal and perinatal morbidity and mortality. Transient neurological deficit is common but persistent deficits are rare. Renal failure complicating eclampsia may result in prolonged renal insufficiency. Hepatic dysfunction is a result of associated liver parenchymal damage, periportal necrosis and rarely, hepatic rupture. Pre-eclampsia has been shown to be associated with diastolic dysfunction, increase cardiac work, and left ventricular indices with evidence of myocardial damage. Cerebrovascular accidents are common; in the long term cardiac and metabolic disease risks are increased. In the fetus pre-term delivery, asphyxia and intra-uterine growth restrictions commonly associated with the disease increase the perinatal mortality. Pregnancy related complications like abruption placentae, HELLP syndrome are frequent associations and pose a risk to both mother and fetus. Pre-eclampsia and eclampsia are independent risk factors for cerebral palsy. Perinatal mortality is increased and neonatal intensive care admission is common. In this study, we analyzed consecutive cases of eclampsia to determine the maternal and fetal outcomes and discuss ways to improve them.

\section{Methods}

This is a prospective study of patients admitted with eclampsia and managed in the maternity unit of Siddhartha Medical College, Govt. General Hospital, Vijayawada, Andhra Pradesh. Eclampsia was diagnosed when convulsions occur in association with syndrome of pre-eclampsia in the second half of pregnancy and the puerperium in patients without known history of convulsions, meningitis, or head injury. All patients were screened for malaria on admission using peripheral blood film to exclude cerebral malaria. Patients with clinical diagnosis of cerebral malaria and other medical causes of convulsion and coma were excluded. Data were prospectively collected and information recorded includes obstetrics characteristics, gestational age, mode of 
delivery, intensive care unit admission, time of eclampsia in relation to labour and maternal and fetal complications. All patients were followed up from admission with eclamptic convulsions through to discharge, for the duration of their hospital stay.

\begin{tabular}{lll}
\hline Gravida & No. of cases & Percentage \\
\hline Primi Gravidae & 58 & $78.3 \%$ \\
\hline $\mathbf{2}^{\text {nd }}$ Gravidae & 12 & $16.2 \%$ \\
$\mathbf{3}^{\text {rd }}$ Gravidae & 4 & $5.4 \%$ \\
\hline
\end{tabular}

\section{Result}

Seventy four consecutive cases of eclampsia were recorded out of 6880 deliveries, giving an incidence of 10.7 per 1000 deliveries. Fourteen $(18.9 \%)$ of the cases had antenatal care in our hospital. While $60(81.08 \%)$ gave no account of antenatal care in this hospital. They are referred from various PHC, CHC, AH and District hospitals.

Table-1Common age group is $-18-23$ years $=72.9 \%$

Table -2 Majority were Primi Gravidae

Table-3 Eclampsia- Relation to Gestational age

\begin{tabular}{lll}
\hline GA & No. of cases & Percentage \\
\hline $\mathbf{2 4 - 2 8}$ weeks & 10 & $13.5 \%$ \\
\hline $\mathbf{2 8 - 3 6}$ weeks & 32 & $43.2 \%$ \\
$\mathbf{3 6 - 4 0}$ weeks & 28 & $37.8 \%$ \\
$\mathbf{\text { 40 weeks }}$ & 4 & $5.4 \%$ \\
\hline
\end{tabular}

Eclampsia common in $3^{\text {rd }}$ trimester of pregnancy

Table-4

\begin{tabular}{|l|l|l|}
\hline Type of eclampsia & No. of cases & Percentage \\
\hline Antepartum eclampsia & 50 & $67.5 \%$ \\
\hline Intrapartum eclampsia & 6 & $8.1 \%$ \\
\hline Postpartum eclampsia & 16 & $21.6 \%$ \\
\hline Intrapartum and postpartum eclampsia & 2 & $2.7 \%$ \\
\hline
\end{tabular}

- 42 cases had severe hypertension on admission

Diastolic BP >110mmHg

- 32 cases had mild hypertension

Diastolic BP 90 to $<110 \mathrm{mmHg}$

Table-5

\begin{tabular}{lll}
\hline Mode of delivery & No. of cases & Percentage \\
\hline LSCS & 40 & $54.05 \%$ \\
\hline Normal vaginal delivery & 25 & $33.3 \%$ \\
Assisted vaginal delivery & 9 & $12 \%$ \\
\hline
\end{tabular}

Majority of cases were delivered by LSCS.

\begin{tabular}{lll}
\hline Age & Number & Percentage \\
\hline $\mathbf{1 8 - 2 3}$ yrs & 54 & $72.9 \%$ \\
$\mathbf{2 3 - 2 7}$ yrs & 15 & $20.2 \%$ \\
\hline $\mathbf{2 7}$ yrs & 5 & $6.7 \%$ \\
\hline
\end{tabular}

Table-6 Maternal complications in eclampsia cases

\begin{tabular}{lll}
\hline Maternal complications & No. of cases & Percentage \\
\hline Prolonged unconsciousness & 2 & \\
& $2.7 \%$ \\
Acute renal failure & 3 & $4.05 \%$ \\
CVA & 2 & $2.7 \%$ \\
HELLP syndrome & 3 & $4.05 \%$ \\
Pulmonary edema & 2 & $2.7 \%$ \\
Coagulation failure & 2 & $2.7 \%$ \\
Abruption & 6 & $8.1 \%$ \\
\hline
\end{tabular}




\begin{tabular}{lcc}
\hline Postpartum haemorrhage & & \\
& 4 & $5.4 \%$ \\
Postpartum psychosis & 2 & $2.7 \%$ \\
Macular edema cortical blindness & 1 & $1.35 \%$ \\
PRES & 1 & $1.35 \%$ \\
Cerebral venous sinus thrombosis & 1 & $1.35 \%$ \\
Death & 2 & $2.7 \%$ \\
\hline
\end{tabular}

Table-6 shows the maternal outcome. Twenty-nine had complications, two had prolonged unconsciousness upto $48 \mathrm{hrs}$, three had acute renal failure, two had CVA, three had HELLP syndrome, two had pulmonary edema, two had coagulation failure, six had abruption, four had PPH, two had postpartum psychosis, one had macular edema cortical blindness, one had PRES and one had cerebral venous sinus thrombosis. There were two maternal deaths. The leading causes of death were pulmonary edema and acute renal failure.

Table-7 - Perinatal complications in eclampsia cases

\begin{tabular}{lll}
\hline Perinatal complications & No. of cases & Percentage \\
\hline Still births & 12 & $16.2 \%$ \\
\hline Birth asphyxia & 14 & $18.9 \%$ \\
\hline Low birth weight & 16 & $21.6 \%$ \\
\hline Admission in NICU & 10 & $13.5 \%$ \\
\hline
\end{tabular}

The perinatal outcome is shown in table-7. Still births $12(16.2 \%)$, Birth asphyxia 14 (18.9\%), Low birth weight $16(21.6 \%)$ and admission in NICU $10(13.5 \%)$. Most of the NICU admissions were for asphyxia and prematurity.

\section{Discussion}

The incidence of eclampsia in our hospital was 10.7 per 1000 deliveries. A high incidence of eclampsia is common in developing countries where most patients have no antenatal care which would allow for early recognition and treatment of pre-eclampsia. Majority of the patients are young and nulliparous. Most eclamptic seizures occur in the $3^{\text {rd }}$ trimester $86.4 \%$ after 28 weeks of gestation. Antepartum eclampsia - 67.5\%, Postpartum eclampsia - 21.6\%, Intrapartum eclampsia - 8.1\% and Intrapartum \& postpartum eclampsia $2.7 \%$ Complications were recorded in $29(39.1 \%)$ of the patients, with case fatality rate of $2.7 \%$. Coagulopathy complicated $-2.7 \%$ of cases, HELLP syndrome $-4.05 \%$, blood products required to correct them. Nevertheless, their correct identification is important in preventing further bleeding related morbidity during surgery or following trauma of delivery. Six $(8.1 \%)$ of our patients had placental abruption. This is a common cause of renal failure, postpartum haemorrhage and perinatal death. The still birth rate was $16.2 \%$. Hypertensive disorders are a common cause of pre-term labour, perinatal death and intra-uterine growth restrictions. Sixteen patients, $21.6 \%$ had low birth rate. Asphyxia was recorded in $14(18.9 \%)$ of the neonates. NICU admissions are 10 $(13.5 \%)$.

\section{Conclusions}

Eclampsia still remains a major cause of maternal morbidity and mortality. More awareness and enabling factors should be created for more women to access antenatal facilities. Information about danger signs of pre-eclampsia/ eclampsia should be made available to all antenatal cases. 